Résumés des conférences et travaux

\title{
Liste des doctorats soutenus à la section pendant l'année 2009-2010
}

par ordre alphabétique des noms d'auteur

\section{QpenEdition}

\section{Journals}

Édition électronique

URL : https://journals.openedition.org/ashp/1095

DOI : 10.4000/ashp.1095

ISSN : 1969-6310

Éditeur

Publications de l'École Pratique des Hautes Études

Édition imprimée

Date de publication : 1 octobre 2011

Pagination : xiii-xiv

ISSN : 0766-0677

Référence électronique

"Liste des doctorats soutenus à la section pendant l'année 2009-2010 », Annuaire de l'École pratique des hautes études (EPHE), Section des sciences historiques et philologiques [En ligne], 142 | 2011, mis en ligne le 18 juillet 2011, consulté le 06 juillet 2021. URL : http://journals.openedition.org/ashp/1095 ; DOI : https://doi.org/10.4000/ashp.1095 


\section{DIPLÔMES DE L’ÉCOLE SOUTENUS À LA SECTION PENDANT L'ANNÉE 2009-2010

\author{
par ordre alphabétique des noms d'auteur
}

La Vie du roi serbe Étienne de Detchani par Grégoire Camblak en Russie et son intégration dans le cycle des légendes de saint Nicolas (XVI ${ }^{e}-X V I I^{e}$ s.), par $\mathrm{M}^{\mathrm{me}}$ Valérie Geronimi, sous la direction de M. Pierre Gonneau, le 22 mars 2010.

"Acta Alexandrinorum », par M. Chris Rodriguez, sous la direction de M. Joseph MÉLÈze, le 15 décembre 2010.

Les médecins de langue allemande à Paris au XIXe siècle (1803-1871), par M. JeanMarie Mouthon, sous la direction de M. Jacques Le RIDER, le 17 mars 2010.

\section{DOCTORATS SOUTENUS À LA SECTION PENDANT L'ANNÉE 2009-2010}

\section{par ordre alphabétique des noms d'auteur}

Manuscrits de la Genizah à la bibliothèque de l'Alliance israélite universelle : un regard sur la magie hébraïque, par $\mathrm{M}^{\mathrm{me}}$ Emma АвАтЕ, sous la direction de $\mathrm{M}^{\mathrm{me}}$ Judith Olszowy-Schlanger, le 19 juin 2010.

L'histoire du royaume de Byblos à l'âge du fer, 1080-333 av. J.-C., par M. Marc Miled Aвou-Abdallah, sous la direction de M. André Lemaire, le 13 juillet 2010.

La Caisse nationale des monuments historiques, par $\mathrm{M}^{\mathrm{me}}$ Valérie CARPENTIER, sous la direction de M. Jean-Michel Leniaud, le 14 octobre 2010.

Recherches sur les documents séculiers en tokharien : économie bouddhique et société dans la région de Kucha, par $\mathrm{M}^{\mathrm{me}}$ Chao Jung CHING, sous la direction de M. Georges-Jean Pinault, le 25 juin 2010.

Le dialecte de Delphes au IV et au III e s. av. J.-C., par M ${ }^{\text {me }}$ Mathilde DoutheRenard, sous la co-direction de M. Laurent Dubois et de M. Denis Rousset, le 27 mars 2010.

Denys l'Aréopagite et l'origénisme syriaque. Édition critique et étude historique et doctrinale du Traité sur les noms divins dans la version de Serge de Resh'ayna, par Emiliano Bronislaw Fiori, sous la direction de M. Henri HugOnNARD-Roche, le 22 mars 2010.

Histoire culturelle et poétique de la traduction : Alaol et la tradition littéraire bengali au XVII ${ }^{e}$ siècle à Mrauk-U, capitale du royaume d'Arakan, par M. Thibaut D'Hubert, sous la direction de $\mathrm{M}^{\mathrm{me}}$ Françoise Delvoye, le 22 juin 2010.

Mécène en Saxe. Les mécanismes de représentation des favoris à l'exemple d'Heinrich comte de Bruhl, par $\mathrm{M}^{\mathrm{me}}$ Ute Christina $\mathrm{KocH}$, sous la co-direction de M. Michel 
Hochmann et de M. Jurgen Muller (université technique de Dresde), le 22 mars 2010.

Les archives royales du Panduranga : étude des terrains agricoles de la province méridionale du Campa au XVIII ${ }^{e}$ siècle, par $\mathrm{M}^{\mathrm{me}}$ Amandine LePoutre, sous la direction de M. Philippe PAPIN, le 8 mars 2010.

Les conflits de règles à Rome. Validité et valorisation des normes dans la Rome républicaine, par M. Christoph LundgreEn, sous la direction de M. Jean-Louis FERRARY, le 19 décembre 2009.

Histoire du texte de la Batrachomyomachie, des origines aux premiers imprimés, par M. Yann Migoubert, sous la direction de $\mathrm{M}^{\mathrm{me}}$ Brigitte Mondrain, le 5 juin 2010.

Le Jingdu sanmei jing (Sûtra $d u$ samâdhi qui conduit au pur salut) : généalogie d'un " apocryphe » et croyances du bouddhisme populaire dans la Chine des Six Dynasties, par M. Costantino MoretTI, sous la co-direction de M. Jean-Pierre DrÈGE et de $\mathrm{M}^{\mathrm{me}}$ Liying Kuo (EFEO), le 7 mai 2010.

Dispersi sunt lapides sanctuarii. La reconstruction des églises de Meurthe-et-Moselle après la première guerre mondiale (1918-1933), par M. Nicolas PADIOU, sous la direction de M. Jean-Michel Leniaud, le 9 février 2010.

Splendeur du culte et gloire du gouvernement. L'administration des cultes et les pontificaux pendant la période concordataire, par $\mathrm{M}^{\mathrm{me}}$ Maria-Anne PRIVAT, sous la direction de M. Jean-Michel LenIaud, le 15 juin 2010.

Les plombs inscrits de Siscia (Croatie), par M. Ivan RADMAN-LIVAJA, sous la direction de M. Michel ReDDÉ, le 30 janvier 2010.

Le vocabulaire de la mer : étude comparée en latin, grec ancien et indo-aryen ancien, par $\mathrm{M}^{\mathrm{me}}$ Julie SoRBA, sous la direction de M. Georges-Jean Pinault, le 12 février 2010 .

La maison et le temple: les stratégies familiales du clergé durant la première moitié de la XVIII e dynastie, par M. Jean-Christophe Thomazo, sous la direction de M. Pascal Vernus, le 21 janvier 2010.

"Que leurs plaisirs ne finissent jamais ». Spectacles de cour, divertissements et mécénat musical du Grand Siècle aux Lumières : l'exemple des princes de Bourbon-Conti, par M. Thomas Vernet, sous la direction de $\mathrm{M}^{\mathrm{me}}$ Catherine MAssip, le 12 juin 2010.

Diplomatique de l'Église russe (988-1448) : influences byzantines et développements originaux, par M. Konstantinos VetochniKov, sous la direction de M. Pierre GoNNEAU, le 18 mai 2010. 


\section{POST-DOCTORATS SOUTENUS À LA SECTION PENDANT L'ANNÉE 2009-2010} par ordre alphabétique des noms d'auteur

Talio esto. Recherches sur les origines de la formule " cil pour cil, dent pour dent » dans les droits du Proche-Orient ancien, par M. Jan RothкамммI, sous la direction de $\mathrm{M}^{\mathrm{me}}$ Sophie Démare-Lafont, le 14 avril 2010.

\section{HABILITATIONS À LA DIRECTION DE RECHERCHES SOUTENUES À LA SECTION PENDANT L’ANNÉE 2009-2010} par ordre alphabétique des noms d'auteur

Gouverner l'Empire. La nomination des fonctionnaires provinciaux dans l'Empire almohade (1224-1269). Édition, traduction et étude du manuscrit 4752 de la bibliothèque Hasaniyya de Rabat contenant 77 taqādīm (" nominations »), par M. Pascal Buresi, sous la direction de M. François Déroche, le 13 novembre 2009.

Ortus bibliorum avec l'étude inédite Le jour où le soleil s'arrêta. Études de philologie, d'épigraphie et d'histoire du texte de Josué 10, par M. Michael LANGLOIs, sous la direction de M. André LemaIre, le 3 juin 2010.

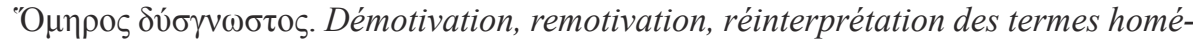
riques à date archaïque et classique, par $\mathrm{M}^{\mathrm{me}}$ Claire LE Feuvre, sous la direction de M. Charles DE Lamberterie, le 20 novembre 2009.

Les dialectes d'Argos et d'Épidaure au contact de l'Attique, puis de la koinè, de l'époque classique à la fin du Haut-Empire, par $\mathrm{M}^{\mathrm{me}}$ Sophie MINON, sous la direction de M. Laurent DuBois, le 21 novembre 2009.

Savoirs et pratiques médicales aux derniers siècles du Moyen Âge, par $\mathrm{M}^{\mathrm{me}}$ Marilyn NICOUD, sous la direction de $\mathrm{M}^{\mathrm{me}}$ Danielle JACQUART, le 28 novembre 2009.

Recherches en philologie hébraïque et araméenne. Échos du passé araméen : témoignages épigraphiques par rapport à la tradition hébraïque et juive des périodes assyrienne et perse (du IX $X^{e}$ au $V^{e}$ siècle avant notre ère), par $\mathrm{M}^{\mathrm{me}}$ Ursula ScHATTNERRIESER, sous la direction de M. André LEMAIRE, le 2 juin 2010.

Bouddhisme chinois et bouddhisme tibétain, spécificité de chaque tradition et interactions, par $\mathrm{M}^{\mathrm{me}}$ Françoise WANG-ToutAIN, sous la direction de M. Jean-Pierre DRÈGE, le 12 juin 2010. 